\title{
Orthogonal Splines in Approximation of Functions
}

\author{
Leontiev V. L. \\ CompMechLab ${ }^{\circledR}$ and Institute of Advanced Manufacturing Technologies at Peter the Great St. Petersburg Poly technic University, \\ Russia
}

Received December 10, 2019; Revised January 27, 2020; Accepted February 18, 2020

Copyright $\bigcirc 2020$ by authors, all rights reserved. Authors agree that this article remains permanently open access under the terms of the Creative Commons Attribution License 4.0 International License

\begin{abstract}
The problem of approximating of a surface given by the values of a function of two arguments in a finite number of points of a certain region in the classical formulation is reduced to solving a system of algebraic equations with tightly filled matrixes or with band matrixes. In the case of complex surfaces, such a problem requires a significant number of arithmetic operations and significant computer time spent on such calculations. The curvilinear boundary of the domain of general type does not allow using classical orthogonal polynomials or trigonometric functions to solve this problem. This paper is devoted to an application of orthogonal splines for creation of approximations of functions in form of finite Fourier series. The orthogonal functions with compact supports give possibilities for creation of such approximations of functions in regions with arbitrary geometry of a boundary in multidimensional cases. A comparis on of the fields of application of classical orthogonal polynomials, trigonometric functions and orthogonal splines in approximation problems is carried out. The advantages of orthogonal splines in multidimensional problems are shown. The formulation of function approximation problem in variational form is given, a systemof equations for coefficients of linear approximation with a diagonal matrix is formed, expressions for Fourier coefficients and approximations in the form of a finite Fourier series are written. Examples of approximations are considered. The efficiency of orthogonal splines is shown. The development of this direction associated with the use of other orthogonalsplines is discussed.
\end{abstract}

Keywords Approximation of Function, Orthogonal Classical Polynomials, Trigonometric Functions, GramSchmidt Procedure, Compactly Supported Functions, Orthogonal Splines, Finite Fourier Series

\section{Introduction}

Solution of approximation problem, how rule, leads to systems of algebraic equations with tightly filled matrixes or with band matrixes.

Compactly supported functions, for example B-splines, can be used for approximation of functions, but it als o leads to systems of algebraic equations with band matrixes. This problem can be solved with help orthogonal compactly supported functions. But the Gram-Schmidt procedure not allows to get systems of orthogonal compactly supported functions. Such procedure not gives possibilities for orthogonalization of compactly supported functions with conservation of compact supports of functions, for example, in the case of spline systems, how was noted in [1]. Franklin functions [2] were generated on the base of the system of Faber functions [3], but compact supports of functions were lost.

The wavelets of Daubechies [4] are first in the world orthogonal compactly supported functions, but these functions not have analytical form, have poor structure, and thus not efficiently used in numerical methods.

The use of orthogonal classical polynomials [5] makes possible to get systems of algebraic equations with diagonal matrixes in problems of approximation of a function of one argument, and gives the approximation of function in a form of a Fourier series. But orthogonal classical polynomials can be used effectively only for approximations of functions of one argument, and also in multidimensional cases for particular variants of domains having a boundary of only a few simple types. Similar comments are related to use of trigonometric functions in approximation problems.

Overcoming these difficulties is possible with the application of orthogonal functions with compact supports. The possibilities and perspectives of such application are described here.

\section{The Orthogonal Splines}

The first in the world orthogonal splines were proposed in $[6,7,8]$ without the Gram-Schmidt procedure of 
orthogonalization. Such orthogonal splines have the analytical form, for example, in the simplest case [8]

$$
\varphi_{i}(x)=\left\{\begin{array}{c}
(\sqrt{2}-1)\left(x_{i-1}-x\right) / h, \\
x \in\left[x_{i-1}, x_{i-1}+h / 2\right] ; \\
(\sqrt{2}+1)\left(x-x_{i}\right) / h+1, \\
x \in\left[x_{i-1}+h / 2, x_{i}\right] ; \\
(\sqrt{2}-1)\left(x-x_{i}\right) / h+1, \\
x \in\left[x_{i}, x_{i}+h / 2\right] ; \\
(\sqrt{2}+1)\left(x_{i+1}-x\right) / h, \\
x \in\left[x_{i}+h / 2, x_{i+1}\right] ; \\
0, x \in\left(-\infty, x_{i-1}\right) \cup\left(x_{i+1},+\infty\right) .
\end{array}\right.
$$

where $h-$ the step of an grid $x_{1}<x_{2}<\cdots<x_{n}$. These functions are orthogonal on every a grid. A grid can be nonuniform and then steps of grid depend on $i$.

These splines are continuous, consist of linear parts, are the generalization of B-splines

$$
\psi_{i}(x)=\left\{\begin{array}{c}
\left(x-x_{i-1}\right) / h, x \in\left[x_{i-1}, x_{i}\right] \\
\left(x_{i+1}-x\right) / h, x \in\left[x_{i}, x_{i+1}\right] \\
0 . x \in\left(-\infty, x_{i-1}\right) \cup\left(x_{i+1},+\infty\right),
\end{array}\right.
$$

which are not orthogonal. The generalization $\varphi_{i}(x)$ was carried [8] out by adding to the B-spline $\psi_{i}(x)$ two similar B-splines, having a compact supports, the sizes $h$ of which in two times less in comparison with the size $2 h$ of the compact support $\left[x_{i-1}, x_{i+1}\right]$ of $\psi_{i}(x)$. This causes the changes of the B-spline graph between nodal points while the values in the nodes are conserved.

The theorem [8] about approximation properties $\varphi_{i}(x)$ show, that

$$
\|f-\tilde{f}\|_{W_{2}^{0}(\boldsymbol{R})} \leq C h\|f\|_{W_{2}^{1}(\boldsymbol{R})},
$$

where $W_{2}^{k},(k=0,1)-$ the Sobolev spaces, C - a constant, $\boldsymbol{R}=(-\infty,+\infty), \tilde{f}-$ the linear approximation connected with $\varphi_{i}(x)$

$$
f(x)=\sum_{i=1}^{n} \alpha_{i} \varphi_{i}(x),
$$

$\alpha_{i}-$ unknown constants.

The approximation of a function of two arguments have next form

$$
\tilde{f}(x, y)=\sum_{i=1}^{n} \sum_{j=N(i)}^{j=M(i)} \alpha_{i j} \varphi_{i}(x) \varphi_{j}(y)
$$

The tensor products of orthogonal splines are used here.

The splines $\varphi_{i}(x)$ are orthogonal, so that

$$
\int_{-\infty}^{+\infty} \varphi_{i}(x) \varphi_{j}(x) d x=\delta_{i j}\left\|\varphi_{i}(x)\right\|_{W_{2}^{0}(\boldsymbol{R})}^{2},
$$

where $\delta_{i j}-$ Kronecker symbols.

\section{The Approximation Problems}

The problem of creation of approximation (1) in 1D case on $[a, b]$ is connected with the functional

$$
J_{1}\left(\alpha_{1}, \alpha_{2}, \ldots, \alpha_{n}\right)=\int_{a}^{b}\left(f(x)-\sum_{i=1}^{n} \alpha_{i} \varphi_{i}(x)\right)^{2} d x .
$$

The stationary condition $\delta J_{1}=0$ gives

$$
\sum_{k=1}^{n} \delta \alpha_{k} \int_{a}^{b}\left(\sum_{i=1}^{n} \alpha_{i} \varphi_{i}-f\right) \varphi_{k} d x=0
$$

and is necessary condition of minimum of $J_{1}$.

The variations $\delta \alpha_{k}$ are linearly independent, consequently, the known equations of least square method

$$
\sum_{i=1}^{n} \alpha_{i} \int_{a}^{b} \varphi_{i} \varphi_{k} d x=\int_{a}^{b} f \varphi_{k} d x, k=1,2, \ldots, n ;
$$

are received. The property (3) allows obtaining Fourier coefficients

$$
\alpha_{k}=\frac{1}{\left\|\varphi_{k}\right\|_{W_{2}^{0}(R)}^{2}} \int_{a}^{b} f \varphi_{k} d x, k=1,2, \ldots, n,
$$

and finite Fourier series (1) takes the form

$$
\tilde{f}(x)=\sum_{i=1}^{n} \frac{\varphi_{i}(x)}{\left\|\varphi_{i}\right\|_{W_{2}^{0}(\boldsymbol{R})}^{2}} \int_{a}^{b} f \varphi_{i} d x .
$$

An approximation has similar form when using the Legendre polynomials or trigonometric functions.

The problem of creation of approximation (2) in 2D case on a domain $\mathrm{S}$ with curvilinear boundary is connected with the functional

$$
J_{2}\left(\alpha_{i j}\right)=\iint_{S}\left(f(x, y)-\sum_{i=1}^{n} \sum_{j=N(i)}^{j=M(i)} \alpha_{i j} \varphi_{i} \varphi_{j}\right)^{2} d S .
$$

The condition of stationary $\delta J_{2}\left(\alpha_{i j}\right)=0$ gives the system of equations

$$
\begin{gathered}
\sum_{i=1}^{n} \sum_{j=N(i)}^{j=M(i)} \alpha_{i j} \iint_{S} \varphi_{i}(x) \varphi_{j}(y) \varphi_{k}(x) \varphi_{t}(y) d S= \\
=\iint_{S} f(x, y) \varphi_{k}(x) \varphi_{t}(y) d S, \\
k=1,2, \ldots, n ; t=N(k), N(k)+1, \ldots, M(k) .
\end{gathered}
$$

The matrix of this system is diagonal with account (3). The solution of the system(7) gives the Fourier coefficients

$$
\alpha_{i j}=\frac{1}{\left\|\varphi_{i}\right\|_{W_{2}^{0}}^{2}\left\|\varphi_{j}\right\|_{W_{2}^{0}}^{2}} \iint_{S} f \varphi_{i}(x) \varphi_{j}(y) d S
$$

for all nodes of grid. The approximation (2) takes the form of finite Fourier series

$$
\tilde{f}=\sum_{i=1}^{n} \sum_{j=N(i)}^{j=M(i)} \frac{\varphi_{i}(x) \varphi_{j}(y)}{\left\|\varphi_{i}\right\|_{W_{2}^{0}}^{2}\left\|\varphi_{j}\right\|_{W_{2}^{0}}^{2}} \iint_{S} f \varphi_{i} \varphi_{j} d S .
$$

Similar form of an approximation is impossible in the case arbitrary boundary of domain $S$ if use the Legendre polynomials or trigonometric functions. The orthogonal 
splines give the approximations (8) in the case arbitrary boundary of domain $S$. Thus, the solutions of the problem of approximation have the forms (6), (8). The necessity of solution of systems (4), (7) is absent. The amount of computational time is greatly reduced. This is of great importance in the construction of geometric models of complex structures.

The square of the norm of the functions $\varphi_{k}$ has the form

$$
\left\|\varphi_{k}\right\|_{W_{2}^{0}(\boldsymbol{R})}^{2}=\int_{a}^{b} \varphi_{k}^{2}(x) d x=I_{1 i}+I_{2 i}+I_{3 i}+I_{4 i},
$$

where

$$
\begin{gathered}
I_{1 i}=\int_{x_{i-1}}^{x_{i-1}+\frac{h}{2}}\left[\frac{(\sqrt{2}-1)\left(x_{i-1}-x\right)}{h}\right]^{2} d x, \\
I_{2 i}=\int_{x_{i-1}+\frac{h}{2}}^{x_{i}}\left[\frac{(\sqrt{2}+1)\left(x-x_{i}\right)}{h}+1\right]^{2} d x, \\
I_{3 i}=\int_{x_{i}}^{x_{i}+\frac{h}{2}}\left[\frac{(\sqrt{2}-1)\left(x-x_{i}\right)}{h}+1\right]^{2} d x \\
I_{4 i}=\int_{x_{i+1}}^{h}\left[\frac{(\sqrt{2}+1)\left(x_{i+1}-x\right)}{h}\right]^{2} d x .
\end{gathered}
$$

The scalar product of the approximated function and the basis grid function is written as

$$
\int_{a}^{b} f \varphi_{k} d x=\left(f, \varphi_{k}\right)=I_{5 i}+I_{6 i}+I_{7 i}+I_{8 i},
$$

where

$$
\begin{gathered}
I_{5 i}=\int_{x_{i-1}}^{x_{i-1}+\frac{h}{2}}\left[\frac{(\sqrt{2}-1)\left(x_{i-1}-x\right)}{h}\right] f(x) d x, \\
I_{6 i}=\int_{x_{i-1}+\frac{h}{2}}^{x_{i}}\left[\frac{(\sqrt{2}+1)\left(x-x_{i}\right)}{h}+1\right] f(x) d x, \\
I_{7 i}=\int_{x_{i}}^{x_{i}+\frac{h}{2}}\left[\frac{(\sqrt{2}-1)\left(x-x_{i}\right)}{h}+1\right] f(x) d x, \\
I_{8 i}=\int_{x_{i+1}}^{h}\left[\frac{(\sqrt{2}+1)\left(x_{i+1}-x\right)}{h}\right] f(x) d x .
\end{gathered}
$$

\section{The Results}

1. The problem of approximation of the function $f(x)=x^{3}$ on the segment $[-1,0]$ is considered.
The formula (5) with account the formulas (9), (10) gives the values of the coefficients $\alpha_{i}$ for different number of grid nodes. Table 1 contains such values. The integrals were found using the program Maple in the simple analytical form. For example,

$$
I_{1 i}=(\sqrt{2}-1)^{2} h / 24, \quad I_{2 i}=(3 / 8-\sqrt{2} / 6) h .
$$

The values of the integrals were calculated so that the 20 digits in the mantissa were taken into account.

Table 1. Fourier coefficients

\begin{tabular}{|c|l|l|c|}
\hline \multirow{2}{*}{$n$} & \multicolumn{3}{|c|}{$\alpha_{i}$} \\
\cline { 2 - 4 } & $\begin{array}{l}x_{i}=-1 \quad(i \\
=1)\end{array}$ & \multicolumn{2}{|c|}{$x_{i}=-0.5$} \\
\hline 21 & -0.9412664 & -0.1123502 & $i=11$ \\
\hline 51 & -0.9761189 & -0.1197956 & $i=26$ \\
\hline 101 & -0.9879921 & -0.1223732 & $i=51$ \\
\hline 201 & -0.9939808 & -0.1236804 & $i=101$ \\
\hline 401 & -0.9969863 & -0.1243387 & $i=201$ \\
\hline 501 & -0.9975884 & -0.1244707 & $i=251$ \\
\hline 1001 & -0.9987936 & -0.1247351 & $i=501$ \\
\hline
\end{tabular}

The exact values of the function $f(x)=x^{3}$ are taken in nodes, which are used in Table 1:

$$
f(-1)=-1, \quad f(-0.5)=-0.125 \text {. }
$$

The linear approximation (1) uses continuous orthogonal splines $\varphi_{i}(x)$, which consist of linear parts. Thus, the values of the coefficients $\alpha_{i}$ are the values of the linear approximation (1) in the nodes $x_{i}$.

Comparing the values of the coefficients $\alpha_{i}$ from Table 1 with the exact values of the function shows a decrease in the approximation errors with an increase in the number of grid nodes. The dependence of the approximation errors $\varepsilon_{1}, \varepsilon_{2}$ at the two selected points on the number $n$ of grid nodes shows on Fig. 1 the character of convergence of the approximation sequence to the function $f(x)=x^{3}$. Fig. 1 describes the approximation errors $\varepsilon_{k}[\%](k=1,2)$ which refer to the corresponding nodes

$$
x_{1}=-1, \quad x_{2}=-0.5 \text {. }
$$

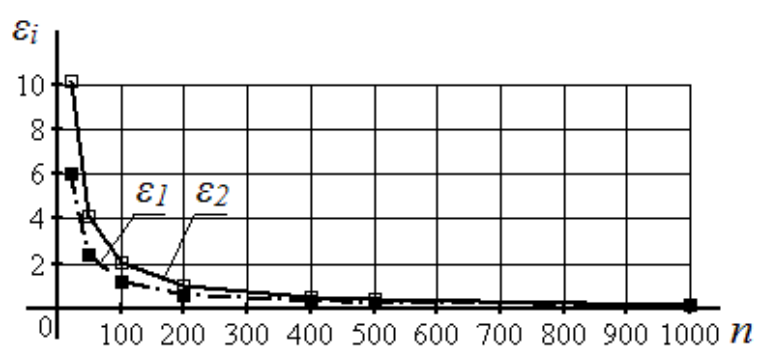

Figure 1. The approximation errors

The approximation error $\varepsilon_{1}$ equals to $0.24 \%$ for $n=501$ 
and $0.13 \%$ for $n=1001$. The approximation error $\varepsilon_{2}$ equals to $0.42 \%$ for $n=501$ and $0.21 \%$ for $n=1001$.

After calculating the Fourier coefficients, a linear approximation of the function is found. The approximation has the form of a finite Fourier series

$$
f(x)=\sum_{i=1}^{n} \alpha_{i} \varphi_{i}(x) .
$$

Increase of the number of nodes causes a decrease in the variations of the straight lines of the graph of the approximation function between nodes caused by the modification of $\psi_{i}(x)$ in the transition to $\varphi_{i}(x)$ [8], but for a small number of nodes, the effect of such deviations between nodes can be noticeable. It is proposed to replace functions $\varphi_{i}(x)$ on functions $\psi_{i}(x)$ after the definition of the function $\tilde{f}(x)$, while retaining the values of all coefficients. It gives the approximation

$$
f(x)=\sum_{i=1}^{n} \alpha_{i} \psi_{i}(x)=\sum_{i=1}^{n} \frac{\psi_{i}(x)}{\left\|\varphi_{i}\right\|_{W_{2}^{0}(R)}^{2}} \int_{a}^{b} f \varphi_{i} d x,
$$

where auxiliary B-splines, added for modification of Bsplines $\psi_{i}(x)$, are omitted. The nodal values of the approximations $f(x), f(x)$ are same. The results show that the convergence of the sequences of the approximations $f(x), f(x)$ is uniform.

2. The problem of approximation of the function $f(x, y)=x^{3} y^{3}$ is considered on a $2 \mathrm{D}$ region, which boundary is, for example, circle with radius equals two.

The exact values of the function $f(x, y)=x^{3} y^{3}$ in taken nodes $\left(x_{i}, y_{j}\right)$ :

$$
f(-1,-1)=1, f(-0.5,-0.5)=0.015625 .
$$

Comparing the values of the coefficients $\alpha_{i j}$ from Table 2 with the exact values of the function shows a decrease in the approximation errors with an increase in the number of grid nodes and with an decrease $h=\delta-$ the

\begin{tabular}{|c|c|c|c|}
\hline \multirow[b]{2}{*}{$h=\delta$} & \multicolumn{3}{|c|}{$\alpha_{i j}$} \\
\hline & $\begin{array}{l}x_{i}=-1, \quad y_{j}=-1, \\
(i, j=1)\end{array}$ & $x_{i}=-0.5$ & $y_{j}=-0.5$ \\
\hline 0.05 & 0.8859824 & 0.0126226 & $i, j=11$ \\
\hline 0.02 & 0.9528081 & 0.0143510 & $i, j=26$ \\
\hline 0.01 & 0.9761283 & 0.0149752 & $i, j=51$ \\
\hline 0.005 & 0.9879978 & 0.0152968 & $i, j=101$ \\
\hline 0.0025 & 0.9939816 & 0.0154601 & $i, j=201$ \\
\hline 0.002 & 0.9951826 & 0.0154930 & $i, j=251$ \\
\hline 0.001 & 0.9975887 & 0.0155588 & $i, j=501$ \\
\hline
\end{tabular}
steps of 2D grids.

Table 2. Fourier coefficients

The dependences on numbers $n=m$ of nodes, taken on the axis $x, y$, the approximation errors $\varepsilon_{3}, \varepsilon_{4}$ [\%] at two selected points $(-1,-1),(-0.5,-0.5)$ are on Fig.2.

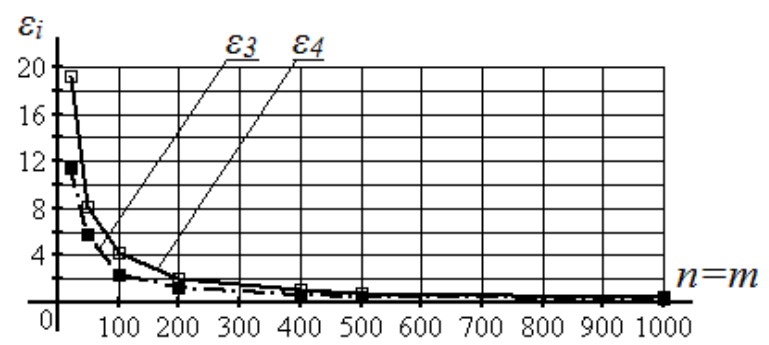

Figure 2. The approximation errors

These dependences show the character of convergence of the approximation sequences to the function $f(x, y)$. On Fig.2, $\varepsilon_{k}[\%](k=3,4)-$ the approximation errors at the corresponding points

$$
\left(x_{1}, y_{1}\right)=(-1,-1), \quad\left(x_{i}, y_{j}\right)=(-0.5,-0.5) .
$$

After calculating the Fourier coefficients $\alpha_{i j}$, a linear approximation of the function is found. The approximation has the form of the finite Fourier series

$$
\tilde{f}(x, y)=\sum_{i=1}^{n} \sum_{j=N(i)}^{j=M(i)} \alpha_{i j} \varphi_{i}(x) \varphi_{j}(y) .
$$

It is proposed to replace functions $\varphi_{i}(x), \varphi_{j}(y)$ on functions $\psi_{i}(x), \psi_{j}(y)$ after the definition of $\tilde{f}(x, y)$, while retaining the values of all coefficients. It gives the approximation

$$
\begin{array}{r}
\hat{f}(x, y)=\sum_{i=1}^{n} \sum_{j=N(i)}^{j=M(i)} \alpha_{i j} \psi_{i}(x) \psi_{j}(y)= \\
\sum_{i=1}^{n} \sum_{j=N(i)}^{j=M(i)} \frac{\psi_{i}(x) \psi_{j}(y)}{\left\|\varphi_{i}\right\|_{W_{2}^{0}}^{2}\left\|\varphi_{j}\right\|_{W_{2}^{0}}^{2}} \iint_{S} f \varphi_{i} \varphi_{j} d S .
\end{array}
$$

The nodal values of the approximations

$$
f(x, y), f(x, y)
$$

are the same. The results show that the convergence of the sequences of these approximations is uniform.

A similar approximation of the function of two arguments can be obtained in the case of another curvilinear boundary of the domain.

\section{Discussion}

With the help of classical orthogonal polynomials or trigonometric functions, approximations similar to the finite Fourier series created in 4.1 can be also obtained. But with the help of such functions, it is impossible in general case to obtain approximations in the form of a finite Fourier series in multidimensional cases. This can be done only for special types of the boundary of the $2 \mathrm{D}$ regions, for example, rectangle or parallelepiped.

Orthogonal splines give possibilities for creation of approximations of functions in regions with arbitrary geometry of a boundary in the form of finite Fourier series in multidimensional cases. Such functions are compactly supported in little areas near corresponding nodes. 
Compact supports create possibility to extract local parts of function. Orthogonality of splines generates diagonal matrix of a system of equations for determination of coefficients of a linear approximation. Orthogonal splines create a base for constructing and developing of universal algorithms of approximation of functions. These algorithms are independent of the shape of the region boundary.

Systems of orthogonal splines of a more general form [8] can also be used for construct finite Fourier series in problems of approximation of functions. The orthogonal splines $\varphi_{i}(x)$ used above are a special case of orthogonal splines of first order of the following form

$$
\phi_{i}(x)=\left\{\begin{array}{c}
\left(x-x_{i-1}\right) / h \\
x \in\left[x_{i-1}, x_{i-1}+h_{1}\right] \cup\left[x_{i-1}+h_{2}, x_{i}\right] \\
-\alpha+2\left(\alpha h+h_{1}\right)\left(x_{i-1}+H-x\right) / \gamma \\
x \in\left[x_{i-1}+h_{1}, x_{i-1}+H\right] ; \\
-\alpha+2\left(\alpha h+h_{2}\right)\left(x-x_{i-1}-H\right) / \gamma \\
x \in\left[x_{i-1}+H, x_{i-1}+h_{2}\right] \\
\left(x_{i+1}-x\right) / h \\
x \in\left[x_{i}, x_{i}+h_{1}\right] \cup\left[x_{i}+h_{2}, x_{i+1}\right] \\
\beta+2\left(\beta h+h_{1}-h\right)\left(x-x_{i}-H\right) / \gamma \\
x \in\left[x_{i}+h_{1}, x_{i}+H\right] \\
\beta+2\left(\beta h+h_{2}-h\right)\left(x_{i}+H-x\right) / \gamma \\
x \in\left[x_{i}+H, x_{i}+h_{2}\right] \\
0, x \in\left(-\infty, x_{i-1}\right) \cup\left(x_{i+1},+\infty\right)
\end{array}\right.
$$

where

$$
\begin{gathered}
\gamma=h\left(h_{2}-h_{1}\right), \\
0 \leq h_{1}<h_{2} \leq h, H=\left(h_{1}+h_{2}\right) / 2, \\
\alpha=\beta-1, h_{1}+h_{2}=h .
\end{gathered}
$$

These conditions provide approximating properties of the functions $\phi_{i}(x)$ and their orthogonality on each grid. if

The functions $\varphi_{i}(x)$ follow from the functions $\phi_{i}(x)$,

$$
\begin{gathered}
h_{1}=0, h_{2}=h, \\
\alpha=\frac{\sqrt{2}-1}{2}, \beta=\frac{\sqrt{2}+1}{2} .
\end{gathered}
$$

Then the conditions of the theorem [8] about approximation properties of orthogonal compactly supported functions are satisfied, thus the functions $\varphi_{i}(x)$ are orthogonal and have approximation properties.

If

$$
\begin{aligned}
& h_{1}=3 h / 8, \quad h_{2}=5 h / 8, \\
& \alpha=\frac{2 \sqrt{2}-1}{2}, \beta=\frac{2 \sqrt{2}+1}{2},
\end{aligned}
$$

then the functions $\phi_{i}(x)$ have approximation properties, because the conditions

$$
h_{1}+h_{2}=h, \quad \alpha=\beta-1
$$

of the theorem [8] are satisfied. These values of $\alpha, \beta$ also provide satisfaction of the condition

$$
4 \alpha \beta+\alpha-\beta-6=0
$$

of orthogonality of these functions on every grid. The functions $\phi_{i}(x)$ have the next form in this other special case

$$
\vartheta_{i}(x)=\left\{\begin{array}{c}
\left(x-x_{i-1}\right) / h \\
x \in\left[x_{i-1}, x_{i-1}+3 h / 8\right] \cup\left[x_{i-1}+5 h / 8, x_{i}\right] \\
-\alpha+8(\alpha+3 / 8)\left(x_{i-1}+h / 2-x\right) / h \\
x \in\left[x_{i-1}+3 h / 8, x_{i-1}+h / 2\right] \\
-\alpha+8(\alpha+5 / 8)\left(x-x_{i-1}-h / 2\right) / h \\
x \in\left[x_{i-1}+h / 2, x_{i-1}+5 h / 8\right] \\
\left(x_{i+1}-x\right) / h \\
x \in\left[x_{i}, x_{i}+3 h / 8\right] \cup\left[x_{i}+5 h / 8, x_{i+1}\right] ; \\
\beta+8(\beta-5 / 8)\left(x-x_{i}-h / 2\right) / h \\
x \in\left[x_{i}+3 h / 8, x_{i}+h / 2\right] \\
\beta+8(\beta-3 / 8)\left(x_{i}+h / 2-x\right) / h \\
x \in\left[x_{i}+h / 2, x_{i}+5 h / 8\right] \\
0, x \in\left(-\infty, x_{i-1}\right) \cup\left(x_{i+1},+\infty\right) .
\end{array}\right.
$$

These functions differ less than the functions $\varphi_{i}(x)$ from non-orthogonal B-splines $\psi_{i}(x)$ and therefore approximations obtained with their help will have higher accuracy compared to $\varphi_{i}(x)$. The use of functions $\vartheta_{i}(x)$ provides additional possibilities for solving approximation problems.

Additional reduction of approximation errors of approximations (11), (12), (13), (14) is as sociated with the use of tens or products of orthogonal splines of the second and third orders [8].

\section{Conclusions}

It is shown that the use of orthogonal splines allows to obtain approximations of functions of many variables in the general case of multidimensional regions having a curvilinear boundary. There are no restrictions on the use of orthogonal splines associated with the shape of the region and the geometry of the boundary. Approximations have the form of finite Fourier series, the construction of which gives a significant saving of computational time. The rationality and efficiency of the approximation algorithm is of great importance in the creation of discrete geometric models for objects with complex surface geometry.

When constructing approximations of the surfaces of technical structures, the coordinates of the selected series of surface points corresponding to both the position of the sensor and the value of some function $f(x, y)$ are determined using measuring devices. Values of integrals in the right parts of the equations of the system(7)

$$
\iint_{S} f(x, y) \varphi_{k}(x) \varphi_{t}(y) d S
$$


are calculated by these coordinates. The solution of the systemof equations (7) is ready in the forms (13) or (14).

The development of this direction is associated with the use of tensor products of orthogonal splines of the second and third orders [8], which are the generalizations of Bsplines. Orthogonal splines [8] of two or three arguments defined on grids consisting of triangles or tetrahedra can be used to reduce errors of approximations of functions on domains with curvilinear boundaries. For example, in the two-dimensional case, such functions are obtained by modifying the Courant functions by analogy with the modification of first-order B-splines.

\section{REFERENCES}

[1] G. Strang, G.J. Fix. An Analysis of the Finite Element Method, Prentige-Hall, Inc, Englewood Cliffs, N.J, 1973.

[2] P. Franklin. A set of continuous orthogonal functions, Mathematische Annalen, 1928, Vol. 100, 522-529.

[3] G. Faber. Über die Orthogonalenfunctionen des Herrn Haar, Jahresber. Deutsch. Math. Verein, 1910, Vol. 19, 104-112.

[4] I. Daubechies. Orthonormal bases of compactly supported wavelets, Commun. Pure Appl. Math., 1988, Vol. 41, No. 7, 909-996.

[5] G. Szego. Orthogonal polynomials, New York City: AMS, 1959.

[6] V.L. Leontiev. Finite element methods based on the use of generalized Courant functions in the theory of elastic oscillations. Abstracts of the all-Russian scientific seminar "Problems of dynamics and strength of electric and power machines", May 18-20, 1993, St. Petersburg: Institute of Problems of Mechanical Engineering of the Russian Academy of Sciences, 21-22. (In Russian).

[7] V.L. Leont'ev, N.C. Lukashanets. Grid Bases of Orthogonal Compactly Supported Functions, Computational Mathematics and Mathematical Physics, 1999, Vol. 39, No. 7, 1116-1126.

[8] V.L. Leontiev. Orthogonal Finite Functions and Numerical Methods, Uly anovsk: State University, 2003. (In Russian). 